\title{
Deep immunity and the progress towards the endemic equilibrium of the Sars-CoV-2.
}

\author{
Samuel Sender $(P h D)^{1}$
}

\begin{abstract}
For airborne viruses, the path to endemicity is characterised by the progression of deep immunity, that is, the decrease in the severity of the virus. Deep immunity cannot be reliably derived from estimates of new cases, since these are not consistently or continuously collected.

We rely on immunological theory to devise a novel indicator of the progression of deep immunity in the active population. This indicator, based on the changing age structure of COVID-related hospitalisations, is more robust than any measures base on serological surveys or PCR tests.
\end{abstract}

We find that at least two-thirds of the path to endemicity has been covered, with a reduction of the severity of the virus of at least two-thirds of the 30-60 years-old population. Measuring the progression of the deep immunity in other age groups is less robust and relevant:

- Virtually the whole under-30 population had acquired deep cross-immunity by exposure to endemic coronaviruses prior to the first wave, so that neither their exposure not the progression of their immunity can be robustly measured

- The immunity of the elderly is not robust, and must be complemented by vaccines, so measuring their exposure is not as relevant).

This indicator and the underlying theory help explaining the recent modification in the characteristics of the epidemy, with higher viral circulation amongst children, higher seasonal circulation and severity, and excess mortality that have recently fallen back to the long-term seasonal trends for all age groups.

Keywords: coronavirus, Sars-CoV-2, Covid, Covid19, estimation, endemicity, immunology, deep immunity JEL codes: C13, C18, C52, I1, I18

\footnotetext{
${ }^{1}$ Associate professor, econometry and finance; consultant. https://www.linkedin.com/in/samuel-sender-phd/ Graphs and estimates in this study are updated here: https://samjs.sjinyapps.io/CoViD

I thank Pierre Sonigo for very rich discussions on the age structure of both immunological memory and immunological strength.

Pierre Sonigo, Chief Scientific Officer of Sebia laboratories https://www.linkedin.com/in/pierre-sonigo/
} 


\section{Table of Contents}

Summary 3

Background $r$

Immunological theory $\quad 3$

Methodology $r$

$\begin{array}{ll}\text { Findings and interpretation } & 4\end{array}$

$\begin{array}{lr}\text { Limitations and alternative data } & 4\end{array}$

Introduction 5

I) From epidemic to endemic: the case of coronaviruses 5

$\begin{array}{ll}\text { A natural transition to endemicity } & 5\end{array}$

What is endemicity for a respiratory virus? $\quad 6$

II) A novel real-time estimation of deep immunity 7

$\begin{array}{ll}\text { The unreliability of exposure estimates and need for alternative data } & 7\end{array}$

$\begin{array}{lr}\text { Evidence of age structure } & 8\end{array}$

$\begin{array}{lr}\text { Consistency and bias of the estimator } & 10\end{array}$

$\begin{array}{lr}\text { Robustness } & 10\end{array}$

III) Long-term trends: limitations and alternative data 11

Limitations: hospital data $r$

$\begin{array}{ll}\text { Long-term mortality trends } & 11\end{array}$

$\begin{array}{ll}\text { Conclusion } & 12\end{array}$

Appendice: Seasonality of airborne viruses and Sars-CoV-2 13

References 14 


\section{Summary}

\section{Background}

Flu- and cold-type airborne viruses generally do not disappear, but co-evolve with the immune system of their hosts, and acquire stable properties in the so-called endemic phase.

For airborne viruses, the path to endemicity is characterised by the progression of deep immunity, that is, the decrease in the severity of the virus.

Ideally, the time varying severity would be computed by the ratio of new severe cases (hospitalisation, or death) to the ratio of new cases (infections), provided these are reliable and continuously collected.

However, estimates of infections are not reliable: PCR tests are subject to participation bias, and serological surveys are not frequently repeated; both measures are biased.

\section{Immunological theory}

Immunological theory can be used to describe the path to endemicity:

- for all new viruses, the early epidemic phase is characterised by a high degree of severe infections; while some viruses can be virtually eliminated, the airborne viruses such as the flu and common eventually reach an endemic phase characterised by high deep immunity in the population which makes infection socially acceptable but does not prevent the circulation of the virus.

- the endemic phase in airborne viruses is characterised by an age structure of circulation and casualties:

o circulation is intense amongst children (with occasional severe forms with toddlers)

$\circ$ the working age population has acquired a high level of immunity through repeated exposure

○ vaccines supplement the declining and eventually defective immune system of the fragile and elderly population, but the older population represent the bulk of hospitalisations and casualties

To sum things up, in the early epidemic phase a new virus can provoke severe diseases at all ages; in the endemic phase, it mostly impacts the fragile, elderly population. The transition to endemicity can be described by a progressive steepening of the age structure of hospitalisations and casualties.

\section{Methodology}

In some countries such as France and the United States, the age structure of severe forms is freely available, with daily updated hospitalisation data.

We build and instantaneous indicator of the relative severity of the virus, which tracks the evolution of the age structure of hospitalisations, namely the ratio of the 30-60 years-old to the ratio of the $60+$ years-old hospitalised - taking into account the higher vaccinal immunity starting in 2021. 


\section{Findings and interpretation}

We find that deep immunity has progressed by more than two thirds in the working-age population; this implies not only that the vast majority of the population has been exposed to the Sars-CoV-2, but also that the new variants did not (so far) massively escape acquired deep immunity.

New variants seem to prevent further overall strengthening of the acquired deep immunity; vaccination seems to have benefited the older generation.

\section{Limitations and alternative data}

Long-term hospitalisation data is needed to assess how much the current epidemic wave deviates from seasonal patterns of known flu- and cold-type viruses to which endemic coronaviruses belong.

The only alternative long-term measure is the all-causes mortality. Long-term mortality data however has fallen back to long-term seasonal trends for all age groups.

This can be interpreted as the joint result of the rise in acquired immunity for the working age population and of the rise in vaccinal immunity for the old-age population.

Mortality data thus suggests a progressive transition to an endemic phase in which the SarsCoV-2 would have similar impact to that of other airborne viruses. 


\section{Introduction}

One year into the epidemic, political, business and personal planning requires assessing longterm prospects. It is important not only to try to assess how far we are from the endemic phase (the new normal), but also to try to characterise it with the first indications at hand : are we heading towards an endless crisis, towards a world with permanent partial confinement, with stop and go policies, or will the Sars-CoV-2 assimilate to our lives just as the seasonal flu and the four endemic coronaviruses its endemic cousins, with similar, acceptable impact on our health systems and daily lives?

Acquired deep immunity acts as a buffer and makes new contaminations less deadly; without deep immunity, more contagious variants could have devastating consequences; with deep immunity, they may progressively lead to the type of seasonal waves known for airborne viruses.

We then seek to measure more directly the progression of deep immunity in the population; we propose a statistically robust approach based on age structure of hospital data.

We find that two-thirds of the active population not previously immunised have already acquired deep immunity, which makes the risk of an explosion in deaths rates not quite likely.

\section{I) From epidemic to endemic: the case of coronaviruses \\ An immunological look at the epidemiological models}

A natural transition to endemicity

Viruses have intrinsic characteristics; however, the pathogenicity of a virus is not an intrinsic characteristic of the virus but of its relationship to the host.

- Virus intrinsic characteristic can be related to their ability to be easily transmitted (this is the case of respiratory viruses), their ability to attach to cell receptors (this is the case of Sars-CoV-2 with the Spike protein on ACE2 receptors), their ability to multiply in certain organs or cell types, and then their ability to damage these organs, either directly (cell degradation) or indirectly (inflammation, action of immune system).

- Pathogenicity is the joint result of the intrinsic characteristics of the virus and the immune defences acquired by first exposure to the virus (so-called homologous acquired immunity), by exposure to closely related viruses (cross-acquired immunity), or by vaccination (vaccine immunity).

For airborne viruses with a very high potential for circulation, there majority of the population tends to be naturally exposed to the virus; this exposure in turn allows the building of deep immune defences in the population.

It is important to note that in virology, vaccines never provide better immunity than the infection itself. It is therefore mostly unnecessary to vaccinate people who are already infected. The more recent the infection or vaccination, and the less different the first infecting virus is from the one that arrives later, the greater the immune protection.

For example, infection with endemic coronaviruses, which occurs in virtually $100 \%$ of the population in childhood around the age of 3 , provides very good protection against Sars-CoV- 
2, despite the strong difference between the immunising virus and the virus that comes second, during the decade that follows, i.e., children and young subjects.

This protection becomes insufficient with advancing age: in addition to the natural decline in immunity acquired over time, there is a difference between the first and second virus.

\section{What is endemicity for a respiratory virus?}

For many viruses that do not affect the respiratory tract, immunisation of an individual and elimination of the virus from the body renders that individual non-contagious, and not susceptible to re-infection, thus reducing the circulation of the virus and allowing eradication.

The standard epidemiological model SIR describes eradication by herd immunity: at the beginning of the epidemic, each infected person infects R0 new people; when more than 1$1 / \mathrm{R} 0$ of the population has been immunised and less than 1/R0 is susceptible, each infected person infects less than one person.

For a respiratory virus, there is no such thing as a herd immunity that blocks transmission as for smallpox or polio. It is essential to distinguish between deep immunity, which protects against severe forms affecting the lungs, and mucosal immunity, which blocks transmission. Indeed, mucosal immunity is not very effective, and is transient, compared to deep immunity. It does not prevent the circulation of the virus via the benign upper respiratory tract infection. These are sinusitis or nasopharyngitis, commonly known as colds.

Thus, the population has long been largely immune to serious diseases caused by endemic coronaviruses or influenza, but this does not prevent the virus from circulating via mostly mild infections.

On the question of the duration of protection from past infection, it is essential to distinguish between mild reinfections in the form of colds and infections of deep tissues such as the lung. Protection against colds is poor and mild reinfections occur despite excellent protection against deep infections. Without making this distinction, the question of the duration of immunity or protection against transmission becomes extremely confusing.

The endemic phased is characterised statistically as stationary, i.e., its characteristics are stable (steady state). From a statistical point of view, endemicity can only be determined ex-post, when the passage of years makes it possible to estimate the average and variance of the annual number of deaths.

From a practical point of view, the situation is endemic when the airborne viruses circulate widely in the population (low mucosal immunity) with acceptable effects (strong deep immunity for the vast majority of the population).

This is the case for influenza and 4 coronaviruses (cousins of Sars-CoV-2). Endemic influenza, rhinoviruses and coronaviruses generate a variable number of hospitalisations and deaths each year, but the vast majority of these occur in older people; vaccination of the majority of people over 65 years of age reduces severe forms of influenza $(\mathrm{SpF}, 2000)$, but some years are significantly more severe both globally $(1956-1958,1968-1970)$ and nationally (the years 2009, 2014-15, 2016-17-18 have higher influenza mortality rates) 
From a purely theoretical standpoint, we need to wait twenty years to estimate the mean and annual variance of the pathogenicity of the virus; from a practical point of view, it is necessary to be able to characterise even imperfectly the state of the transition to endemicity and monitor the evolution of pathogenicity through various empirical severity indicators.

\section{II) A novel real-time estimation of deep immunity}

We aim to measure the evolution of global immunity, or more precisely the empirical severity of the virus, which should be an important guide for public policy.

The empirical severity of the virus could be computed by comparing the ratio of new sever forms to a continuously updated measure of new cases.

In the absence of a reliable and continuously updated measures of incidence, data availability and immune logic lead us to use age-specific hospitalisation statistics; in the absence of experimental measures, the most conventional estimation strategy is to use a quasi-experiment regarding quantities of interest, which we will detail further.

The unreliability of exposure estimates and need for alternative data

Monitoring the incidence (also called new cases, infections) would have been very simple if reliable measurements and a statistically sound collection of representative samples had been put in place as for other social or economic phenomena (Sender, 2021).

It would have made it easy to compute not only the proportion of the population already infected by the virus, but also a direct and continuously updated measure of the pathogenicity of the virus.

But the collection methods used so far do not allow for reliable measurement.

- PCR tests are voluntary, and the capacity to test was insufficient before the autumn, so these cannot be used as the basis of a reliable estimate of population exposure.

- Serological surveys are episodic and cannot be used directly for real time monitory of exposures unless one is willing to assume that immunity never increases in the population.

If needed, there is ample evidence that PCR and serological tests are biased:

- Petersen and Phillips (2020) assess that $75 \%$ of the cases were asymptomatic in the first wave, but there are only approximately $50 \%$ of asymptomatic positive in PCR tests.

- The Diamond Princess cruise ship, immobilised for a fortnight in the port of Yokohama in February 2020, with its 3,600 passengers and crew, was able, thanks to the total absence of passenger isolation measures and a common cabin ventilation system, to obtain exposure of all its occupants to the Sars-CoV-2 virus. However, only $20 \%$ of the passengers tested positive.

As is well known and documented in vaccinal recommendation, antibodies build-up progressively after viral exposure; exposure blocked with non-humoural-specific defenses 
(crossed humoural immunity or cell immunity) does not permit the build-up of testable SarsCoV-2 specific antibodies in serological tests.

Ioannidis (2020) studies numerous serological samples and shows the extreme variability of the induced in infection fatality ratios (IFR) estimates, which typically serve as the basis for calculation of population exposure, hence suggesting a lack of robustness. Driscoll et al. (2020) study exposure by age group, and in some regions the population exposure estimates are above $100 \%$.

Evidence of age structure

France, \% of population hospitalised per age group

- 20-29 ans - 30-59 - 60+

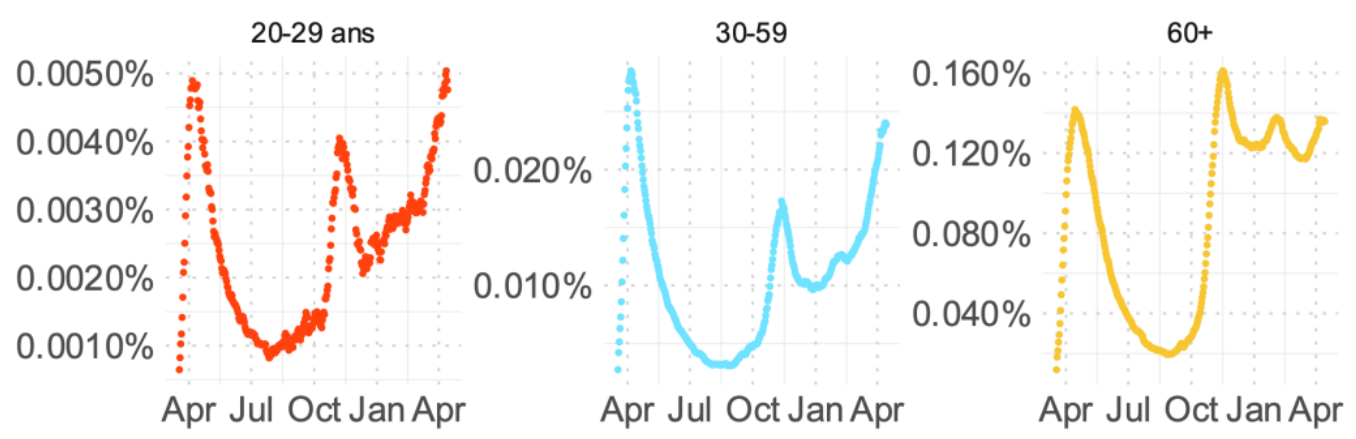

Hospitalisation data show that :

- people under thirty have a deep immunity; the share of the population hospitalised is very weak at the pick of the wave. The natural interpretation is that infection with endemic coronaviruses that occurs in virtually $100 \%$ of the population in childhood around the age of 3 years protects very well against Sars-CoV-2.

- the over-sixties as a group have the weakest immune defences, because of the combined effect of declining acquired immunity and virus mutations.

- the 30-59-year-old population is in an intermediate situation from a statistical point of view ("moderate" fragility); this group will be able to build up immune defences through exposure.

\section{Estimate for France}

The idea is to identify a control population with low immune memory, and a target population with high immune response and memory, but imperfect initial immunisation. Comparing the severe cases in the two populations would then permit to measure the evolution of overall deep immunity in the target population, that is, the reduction in empirical severity given the passage of time and the mutation of Sars-CoV-2.

Our measure of the proportion of people who are immune, or of the evolution of the empirical severity of Sars-CoV-2, is the simple ratio of hospitalisations of 30-59-year-olds to hospitalisations of over 60 -year-olds. 
The data comes from Santé Public France, which benefits from automatic and systematic reporting of Covid cases in hospitals (SI-VIC, victims' identification system), which is made available daily on https://data.gouv.fr

From February 2021, the progressive vaccination of the oldest people weakens our indicator; although it is possible to correct for the vaccination effect, the decrease in the number of frail people makes the indicator less robust and more sensitive to the correction assumptions. In order to eliminate this effect, it is necessary to take into account not the hospitalisations, but the hospitalisations by age group (in relation to the population) reduced by the effective effect of the vaccination.

Vaccination was not carried out randomly but was concentrated on the most vulnerable; the preventive effect of the vaccine and the targeting effect must be taken into account.

At the beginning of April, at least one dose of vaccine was injected into:

- one third of the over-60s

- $\quad$ two-thirds of those over 75

- $\quad 95 \%$ of residents in EHPAD and EMS.

To quantify the targeting effect, we assume that vaccinated people are twice as likely to be at risk as non-vaccinated people, since the probability of a serious COVID event doubles every ten years.

To quantify the vaccine effect, we use the Pfizer (2021) hypothesis that the first injection provides $52 \%$ immunity against severe forms, and the second $92 \%$. We assume a one week delay before protection is effective.

The calculation is done in two steps:

- Let $P_{j}^{V}$ be the population actually vaccinated on day d. We have : $P_{j}^{V}=0.92 \cdot V_{j-7}^{2}+$ $0.52 \cdot\left(V_{j-7}^{1}-V_{j-7}^{2}\right)$ where $V_{j-7}^{1}$ (resp. $\left.V_{j-7}^{2}\right)$ is the cumulative number of people who received a first (resp. second) dose of vaccine at least one week before the date in the age group considered.

- Vaccination reduces the population by a proportion $2 \cdot \frac{P_{j}^{V}}{P+P_{j}^{V}}$ where $\mathrm{P}$ is the population in each major age group.

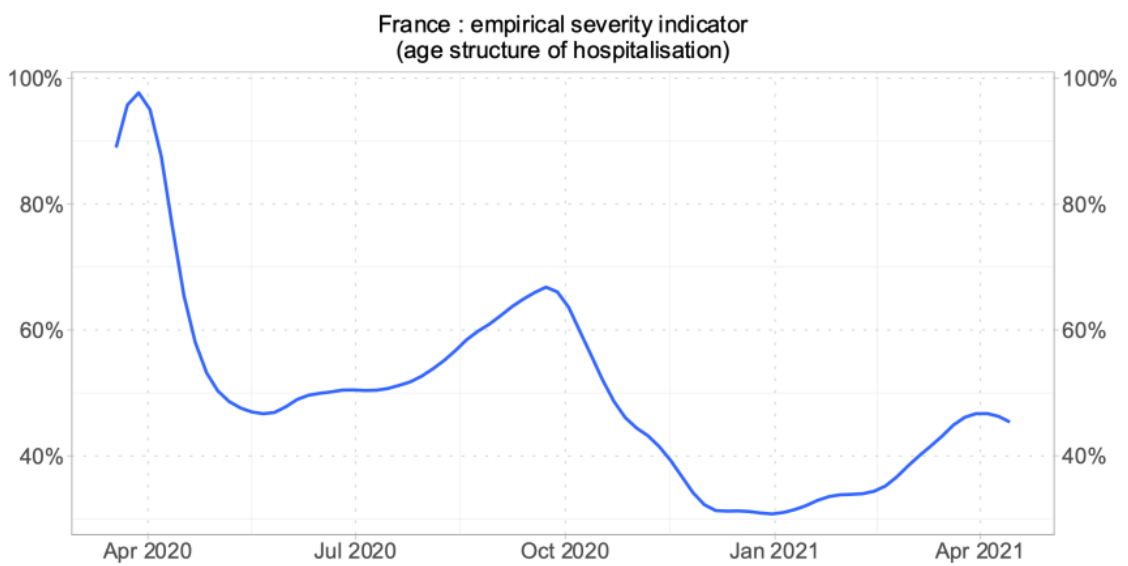

Data source: Santé Publique France, via data.gouv.fr. Authors' calculations. Graphs are updated here: https://samjs.sjinyapps.io/CoViD 
Hospitalization data are more reliable than PCR data and, unlike serological tests, are updated daily. The hypothesis made and the possible changes in data all point towards the possibility of an underestimate of acquired deep immunity:

- The main issue with hospitalisation data is possible classification problems. When it plays, the decrease in severity should logically lead to the categorisation as CoViD patients of an ever-increasing proportion of patients admitted for other pathologies in the 30-59 age group who have built up their immune defences.

- The main issue with our hypothesis is that considering that older people do not acquire immune defences and have not adopted more precautionary behaviour leads to an underestimation of their protection.

These two factors lead to an underestimation of the progress of herd immunity in the adult population, and our estimate of two-thirds is a low bound.

This estimate could be formalised as an estimation problem.

\section{Robustness}

Taking the $75+$ as the fragile reference category leads to a significantly higher estimate of herd immunity in the US (less so in France)

This indicator of the pathogenicity of the virus reflects both the rise in herd immunity and all the factors that influence pathogenicity, such as seasonality and viral mutations.

A moderate increase in severity is also visible during each wave by other indicators, in particular by a rebound in the intensive care (ICU) to hospitalisation ratio that can be observed in each age group.

N.B.: this should be compared with the seasonal variations in admissions to the intensive care unit for other respiratory viruses.

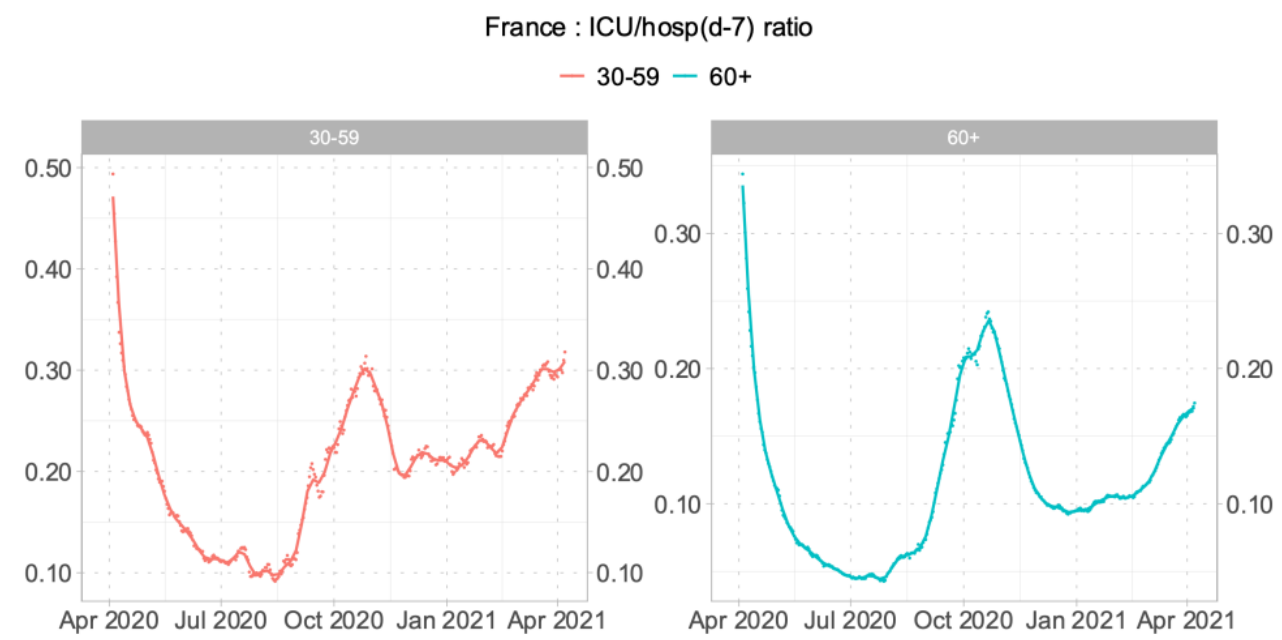




\section{III) Long-term trends: limitations and alternative data}

\section{Limitations: hospital data}

Given the seasonality of the airborne viral infections and given the partial immunological escape of variants in endemic viruses, one cannot expect a 100\% fall in our severity index.

It thus seems important to also examine the hospitalisation patters in light of historical trends: if the Sars-CoV-2 has similar characteristics to other endemic coronaviruses, endemicity may be reached when hospitalisation and mortality exhibit usual patterns.

While this is currently not possible with hospitalisation data, this is partly possible with mortality data.

\section{Long-term mortality trends}

Mortality associated with Covid cases cannot be compared to that associated with other airborne viruses; however, all-causes mortality measures are reliable, a form one possible basis of comparison.

Despite the winter wave of contagion, we have already returned to a historically normal situation:

- INSEE shows that overall mortality since the first of January is almost $10 \%$ lower than in the same period in 2019 for all age categories up to 65 years.

- The z-scores calculated over a longer period by euromomo.eu also show that mortality is now on or below the historical trend for the under-65s since the beginning of the year, and for the over-65s since mid-March and the significant implementation of vaccination.

Euromomo, excess mortality (z-score), 45-64 ans, France

- Z-score … Baseline Normal range … Substantial increase Corrected for delay in registration

France

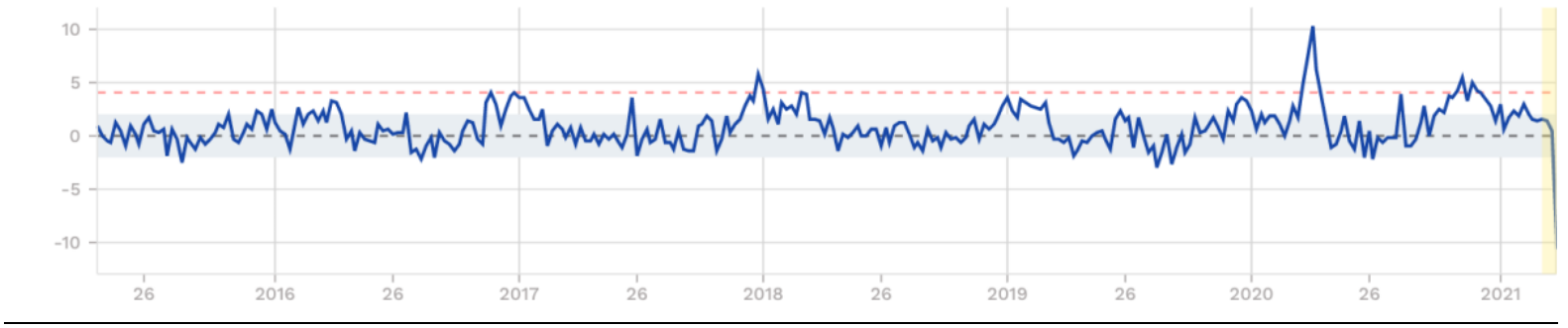

Euromomo, excess mortality (z-score), 65 ans, France 


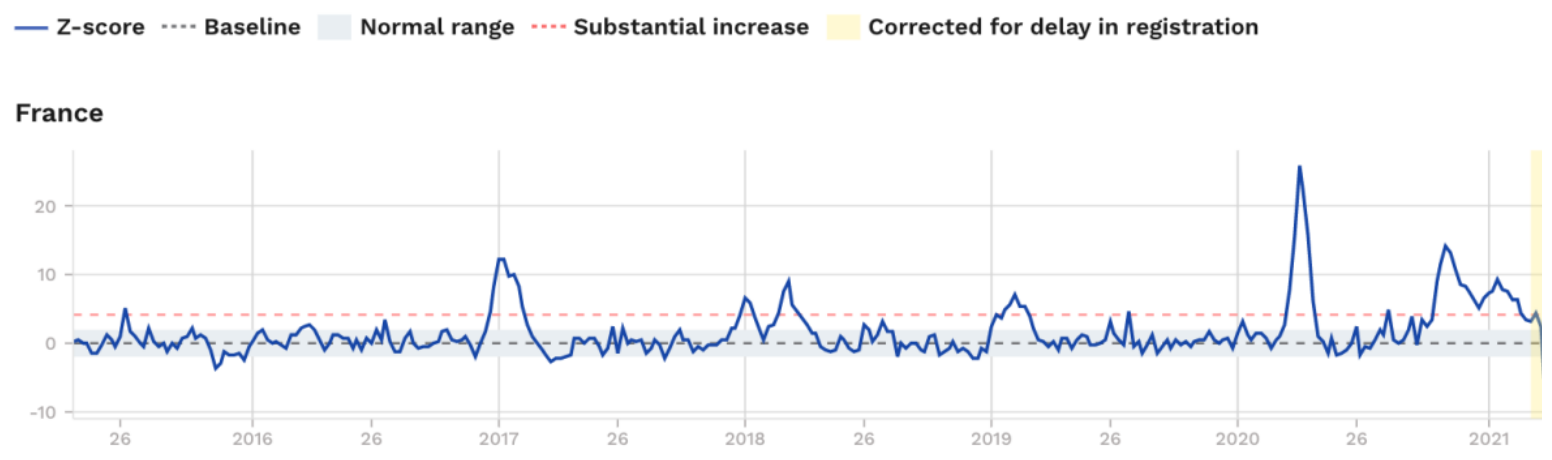

The years 2017, 2018 and 2019 were marked by a winter excess mortality; for 45-64 yearolds, the winter excess mortality is less important at the beginning of 2021 than in 2017-2019; for 65 year-olds and over, the January 2021 peak is similar to that of the years 2017-2019 Source: Euromomo: https://www.euromomo.eu/graphs-and-maps\#z-scores-by-country Methodology : https://www.euromomo.eu/how-it-works/reports-and-publications

Although only the passage of years will tell, these statistics suggest that Sars-CoV-2 could merge with existing coronaviruses without causing significant excess mortality (the alternative being a steady state with higher annual mortality).

\section{Conclusion}

This study presents a new indicator of the progression of deep [herd] immunity in the adult population, which can be calculated in real time in most countries.

The estimate of a two-thirds fall in empirical severity since the first wave suggests an effective progression of deep immunity in the 30-60 age group of the same order of magnitude.

The estimate of the exposure of the under-thirty-year-olds and the elderly is of less practical importance, since the under-thirty-year-olds were in practice immunised before the first wave, and the elderly remain the most fragile and are called upon to be immunised by vaccination.

The weakening of all-causes mortality rates towards long seasonal trends tends support the view of transition to the endemic phase; seasonal increases in severity appear to be emerging.

More data and work would be needed to analyse the determinants of epidemic waves, with their cyclical components, either random or seasonal, and to quantify more precisely the risks of escape from natural and vaccine immunity. 


\section{Appendix: Seasonality of airborne viruses and Sars-CoV-2}

Seasonality is a known phenomenon for all respiratory viruses. Audi et al (2020) show the seasonality of endemic coronaviruses, with atmospheric conditions such as temperature, humidity and oxygen concentration affecting both viral replication and the effectiveness of the immune response (see also DoPico et al, 2005).

In the recent period, the epidemic cycle of COVID corresponds to the classic cycle of typical influenza viruses in our latitudes. The syndromic trends data thus show a very strong decrease in the detections of all the viruses associated with influenza syndromes, with the notable exception of rhinoviruses.

A detailed analysis of these graphs goes beyond the scope of our study. They illustrate that the Sars-CoV-2 circulation surges correspond to the classic seasonal cycle of influenza circulation, from mid-October to mid-April.

\section{Detection of all respiratory viruses}

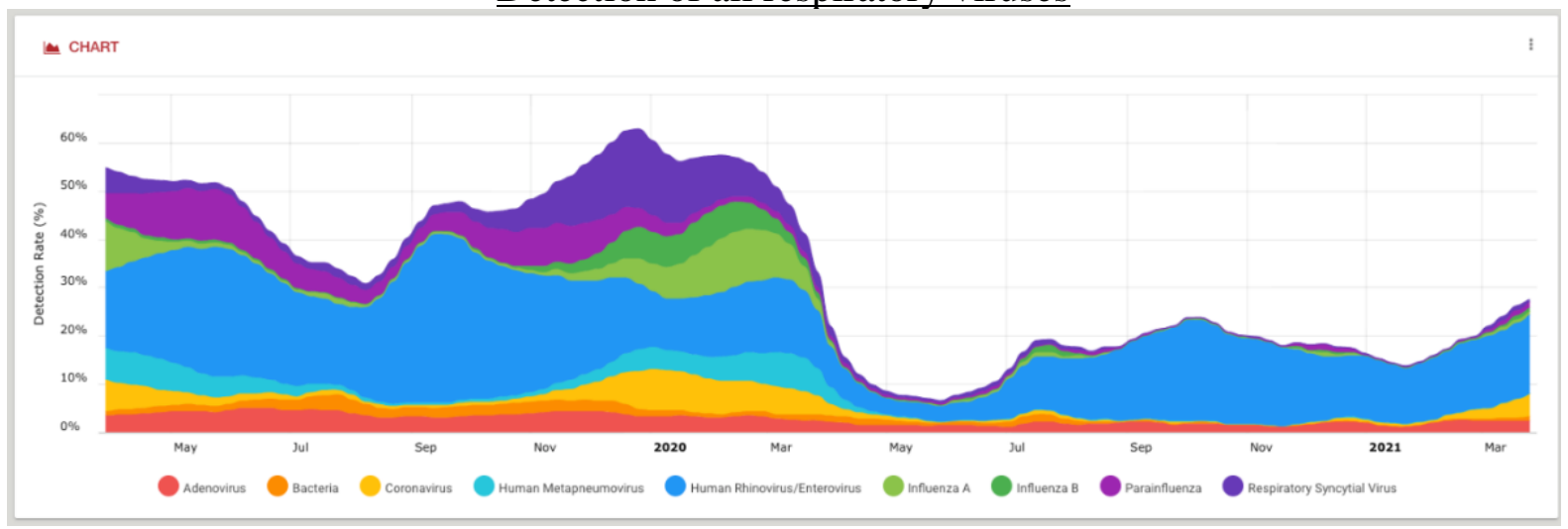

Detection of respiratory viruses, focus on viruses in sharp decline

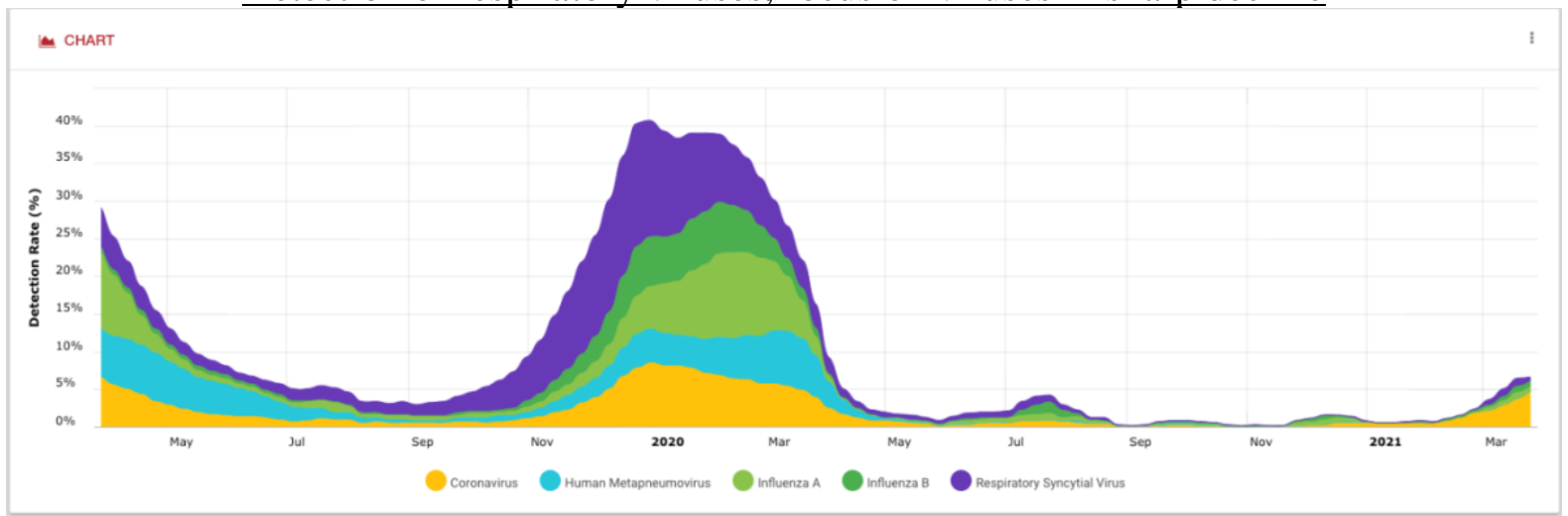

Pathogen detection rates, 3-week centred average, USA.

Source: Syndromic trends. https://syndromictrends.com Methodology: see Meyers et al. (2018) 


\section{References}

Audi et al., 2020. Seasonality of Respiratory Viral Infections: Will COVID-19 Follow Suit? Public Health. https://www.frontiersin.org/articles/10.3389/fpubh.2020.567184/full

Dopico et. al., 2015. Widespread seasonal gene expression reveals annual differences in human immunity and physiology. Nature (6). https://www.nature.com/articles/ncomms8000

Driscoll et al., Nov. 2020. Age-specific mortality and immunity patterns of Sars-CoV-2. Nature (590). https://www.nature.com/articles/s41586-020-2918-0

Ioannidis J, 2020. Infection fatality rate of COVID-19 inferred from seroprevalence data. WHO Bulletin (sept 2020). https://www.who.int/bulletin/volumes/99/1/20-265892/en/

Meyers et al., 2018. Automated Real-Time Collection of Pathogen-Specific Diagnostic Data: Syndromic Infectious Disease Epidemiology. JMIR Journal of Public Health and Surveillance (4-3). https://publichealth.jmir.org/2018/3/e59/

Petersen I., Phillips E., Sept. 2020. Three Quarters of People with Sars-CoV-2 Infection are Asymptomatic: Analysis of English Household Survey Data. Clinical Epidemiology (12). https://www.dovepress.com/three-quarters-of-people-with-sars-cov-2-infection-areasymptomatic-an-peer-reviewed-article-CLEP

Pfizer, 2020. Documentation. https://www.vidal.fr/medicaments/gammes/comirnaty100658.html

Santé Publique France, 2015. Impact de la vaccination contre la grippe saisonnière.

https://www.santepubliquefrance.fr/maladies-et-traumatismes/maladies-et-infectionsrespiratoires/grippe/documents/article/impact-de-la-vaccination-contre-la-grippe-saisonniere-sur-lamortalite-des-personnes-agees-en-france-de-novembre-2000-a-avril-2009

Segondy, M., 2020. Les Coronavirus humains. Rev Francoph Lab. (526). https://www.ncbi.nlm.nih.gov/pmc/articles/PMC7604068/

Sender, S., 2021. Data on a crisis or data crisis ? Linkedin. https://www.linkedin.com/pulse/from-fast-food-data-how-artificial-intelligence-needssender-phd-/

Sonigo, P. 2020. Éléments d'immunologie, Linkedin: https://www.linkedin.com/pulse/comprendre-1\%C3\%A9volution-de-Sars-CoV-2-et-loriginedes-mutants-sonigo/ 